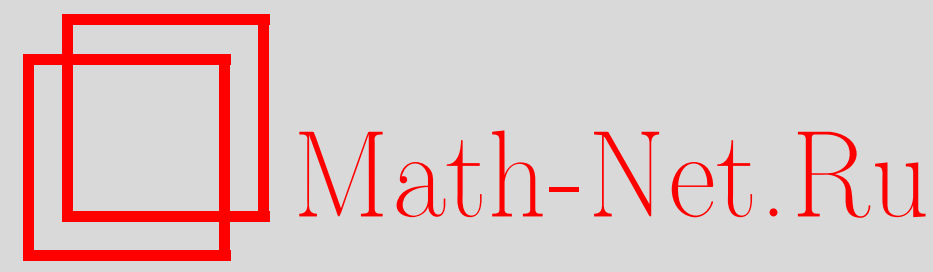

В. В. Щиголев, Конечная базируемость некоторых классов неприводимых представлений симметрических групп, Матем. сб., 2003, том 194, номер 3, 149-160

DOI: https://doi.org/10.4213/sm724

Использование Общероссийского математического портала Math-Net.Ru подразумевает, что вы прочитали и согласны с пользовательским соглашением http://www . mathnet.ru/rus/agreement

Параметры загрузки:

IP : 35.173 .219 .12

26 апреля 2023 г., 16:09:06 
УДК 512.547 .2

\author{
В.В. Щиголев
}

\title{
Конечная базируемость некоторых классов неприводимьх представлений симметрических групп
}

\begin{abstract}
В работе исследуется возможность задания неприводимых представлений симметрических групп при помощи конечного числа соотношений. Доказана конечная базируемость классов представлений, соответствующих разбиениям на две части и разбиениям из фундаментального алькова.

Библиографоия: 11 названий.
\end{abstract}

\section{§1. Введение}

В этой работе мы продолжим исследование, начатое в [1]. Напомним основные использованные там определения. Пусть $X_{n}=\left\{x_{t}^{s}: t \in \mathbb{N}, s=1, \ldots, n\right\}$ - множество коммутируюших переменных, $K$ - поле и $F_{n}=K\left[X_{n}\right]-$ свободная коммутативная алгебра с единицей. Через $F_{n}^{r}$ обозначим подпространство алгебры $F_{n}$, натянутое на мономы вида $x_{i_{1}}^{j_{1}} \cdots x_{i_{r}}^{j_{r}}$, где $\left\{i_{1}, \ldots, i_{r}\right\}=\{1, \ldots, r\}$. Симметрическая группа $G(r)$ степени $r$ действует на $F_{n}^{r}$ слева по формуле $\pi x_{i_{1}}^{j_{1}} \cdots x_{i_{r}}^{j_{r}}=$ $x_{\pi i_{1}}^{j_{1}} \cdots x_{\pi i_{r}}^{j_{r}}$.

Положим $\left[x_{t_{1}}, \ldots, x_{t_{k}}\right]=\sum_{\sigma \in G(k)} \operatorname{sgn}(\sigma) x_{t_{1}}^{\sigma(1)} \cdots x_{t_{k}}^{\sigma(k)}$, где $k=1, \ldots, n$. Пусть $\lambda$ - разбиение числа $r$ на не более чем $n$ частей. Через $S^{\lambda}$ обозначим подпространство в $F_{n}^{r}$, натянутое на многочлены вида $\prod_{i=1}^{\lambda_{1}}\left[x_{t_{i, 1}}, \ldots, x_{t_{i, \mu_{i}}}\right]$, где $\mu$ - разбиение, сопряженное с $\lambda$, и $\left\{t_{i, j}: 1 \leqslant i \leqslant \lambda_{1}, 1 \leqslant j \leqslant \mu_{i}\right\}=\{1, \ldots, r\}$. Для любого натурального $i$ через $\lambda+\left(i^{n}\right)$ обозначим разбиение $\left(\lambda_{1}+i, \ldots, \lambda_{n}+i\right)($ см. $\S 3)$.

Пусть $U$ - некоторый $K G(r)$-подмодуль модуля $F_{n}^{r}$. Через $U \uparrow$ (или более точно через $U \uparrow_{n}$, если нужно явно указать $\left.n\right)$ мы обозначим $K G(r+n)$-подмодуль модуля $F_{n}^{r+n}$, порожденный подпространством $U\left[x_{r+1}, \ldots, x_{r+n}\right]$. Заметим, что если $U \subset S^{\lambda}$, то $U \uparrow \subset S^{\lambda+\left(1^{n}\right)}$.

Обратно, пусть $V$ - некоторый $K G(r+n)$-подмодуль модуля $F_{n}^{r+n}$. Через $V \downarrow$ (или через $V \downarrow_{n}$ ) мы обозначим множество таких многочленов $f \in F_{n}^{r}$, что $f\left[x_{r+1}, \ldots, x_{r+n}\right] \in V$. Понятно, что $V \downarrow$ есть $K G(r)$-модуль. С помошью следствия 17.18 работы [2] легко доказать, что если $V \subset S^{\lambda+\left(1^{n}\right)}$, то $V \downarrow \subset S^{\lambda}$.

Через $U \uparrow^{t}$ или $U \downarrow^{t}$ будем обозначать результат $t$ кратного применения к $U$ операции $\uparrow$ или $\downarrow$ соответственно, конечно, если это возможно (для $\left.U \downarrow^{t}\right)$. Отметим следуюшие простые свойства: $U \downarrow \uparrow \subset U \subset U \uparrow \downarrow$ и $U \downarrow \uparrow \downarrow=U \downarrow$ для любого подмодуля $U$ модуля $F_{n}^{r}$, где $r \geqslant n$.

В работе [1] была сформулирована следуюшая 
ПроБлемА 2. Пусть $V_{i} \subset S^{\lambda+\left(q_{i}^{n}\right)}, i \in \mathbb{N}$, әде $0 \leqslant q_{1}<q_{2}<\cdots$, - некоторая последовательность подмодулей таких, что $V_{i} \uparrow^{q_{i+1}-q_{i}} \subset V_{i+1}$ для каждого $i \in \mathbb{N}$. Существует ли такое число $N$, что для кажсдого $i>N$ выполнено $V_{N} \uparrow q_{i}-q_{N}=V_{i}$ ?

Эта проблема рассматривалась как некоторый частный, но важньй случай следующей проблемы.

ПроБЛема 1. Предположим, что сhar $K=p>0$. Верно ли, что любая последовательность многочленов алгебры А конечно базируема?

Здесь $A$ - свободная коммутативная $K$-алгебра со свободными порождающими $f_{i}\left(x_{t_{1}}, \ldots, x_{t_{n(i)}}\right)$, где $i=1, \ldots, k$ и $t_{1}, \ldots, t_{n(i)} \in \mathbb{N}$, полилинейность многочленов понимается относительно кратностей переменных $x_{j}$ (например, многочлен $f\left(x_{1}, x_{2}\right) f\left(x_{2}, x_{3}\right)$ не считается полилинейнњ $)$ и следствие понимается относительно $K$-линейных действий, умножений на элементы из $A$ и переименований переменных $x_{j}$.

Проблему 1 можно рассматривать как аналог проблемы Шпехта для форм. Роль тождеств при этом играют соотношения с формами. Примером таких соотношений могут служить хорошо известные соотношения Гарни. В случае char $K=0$ последние полностью определяют модули Шпехта, а следовательно, и неприводимые модули. В случае char $K=p>0$ это уже неверно и возникает вопрос о существовании конечного количества соотношений, определяющих некоторые классы неприводимых модулей. В силу результатов работы [1] естественно рассматривать классы вида $D^{\lambda+\left(i^{n}\right)}, i \in \mathbb{N} \cup\{0\}$, где $\lambda+\left(1^{n}\right)-p$-регулярное разбиение. Такой выбор соответствует решению проблемы 2 в случае, когда $q_{i}=i-1$ и $V_{i}$-максимальный подмодуль модуля $S^{\lambda+\left(i-1^{n}\right)}$ для каждого $i \in \mathbb{N}$.

Положительное решение последней проблемы для тех разбиений $\lambda$, для которых выполнено условие 1 , сформулированное далее в $\S 3$, получено в теореме 1 . В силу результатов работ [3] и [4] существуют разбиения $\lambda$, для которых условие 1 не выполнено. Однако точно известно, что оно выполнено для разбиений $\lambda$, содержащих не более $n$ ненулевых частей и удовлетворяющих неравенству $\lambda_{1}-\lambda_{n} \leqslant p-n$, и для разбиений, состоящих из двух частей. Проверка этого произведена в $\S 4$ на основании результатов работ [5] и [6]. Заметим, что случай $n=2$ рассмотрен просто для примера, так как теорема 6 работы [1] решает частный случай $n=2$ проблемы 1 без каких-либо ограничений на подмодули $V_{i}$.

\section{§2. Предварительные сведения}

Фиксируем алгебраически замкнутое поле $K$ положительной характеристики $p$ и натуральное число $n \geqslant 2$. Пусть $E$ - линейное пространство над $K$ с базиcoм $v_{1}, \ldots, v_{n}$ и $G L_{n}(K)$ - группа всех $K$-линейных автоморфизмов пространства $E$. Группа $G L_{n}(K)$ естественным образом снабжается структурой алгебраической $K$-группы, и мы можем рассматривать полиномиальные (рациональные) $G L_{n}(K)$-модули относительно этой структуры. Через $\Lambda$ обозначим множество всех наборов целых чисел длины $n$. Будем писать $\lambda \unrhd \mu$, где $\lambda, \mu \in \Lambda$, если для любого $i=1, \ldots, n$ выполнено $\lambda_{1}+\cdots+\lambda_{i} \geqslant \mu_{1}+\cdots+\mu_{i}$. Если $V$ - полиномиальный $G L_{n}(K)$-модуль и $\lambda=\left(\lambda_{1}, \ldots, \lambda_{n}\right)$ - набор целых чисел, то через $V^{\lambda}$ обо- 
значим множество векторов $v \in V$ таких, что $\operatorname{diag}\left(t_{1}, \ldots, t_{n}\right) v=t_{1}^{\lambda_{1}} \cdots t_{n}^{\lambda_{n}} v$ для любых $t_{1}, \ldots, t_{n} \in K^{*}$. Очевидно, что $V^{\lambda}$ - линейное пространство над $K$. Это пространство называется весовым пространством модуля $V$, соответствующим $\lambda$. В случае $V^{\lambda} \neq 0$ набор $\lambda$ назьвается весом модуля $V$. Через $\Lambda^{+}$обозначим подмножество множества $\Lambda$, состоящее из наборов $\left(\lambda_{1}, \ldots, \lambda_{n}\right)$ таких, что $\lambda_{1} \geqslant \cdots \geqslant \lambda_{n}$

В работах [7] и [8] развита теория так называемых наклонных модулей ("tilting modules"). В этой работе мы считаем, что любой наклонньй модуль есть конечномерный полиномиальный $G L_{n}(K)$-модуль. Мы не будем давать определения этих объектов. Вместо этого перечислим их основные свойства, необходимые для дальнейшего изложения.

1) Модуль $E$ наклонный (в силу определений работ [7] и [8]).

2) Тензорное произведение наклонных модулей - наклоннњй модуль (первое полное доказательство в общем случае получено О. Матье в [9]).

3) Для любого $\lambda \in \Lambda^{+}$сушествует неразложимый наклонный модуль $P(\lambda)$ такой, что $\operatorname{dim} P(\lambda)^{\lambda}=1$ и, если для некоторого $\mu \in \Lambda$ выполнено $\operatorname{dim} P(\lambda)^{\mu}>0$, то $\mu \unlhd \lambda$ (см. [7; теорема (1.1)]).

4) Любой наклонньй модуль разлагается в прямую сумму наклонных модулей, изоморфных $P(\lambda), \lambda \in \Lambda^{+}$(см. [7; теорема (1.1)]).

Для любого натурального $r$ через $G(r)$ обозначим симметрическую группу степени $r$, т.е. множество таких биекций $\sigma$ множества натуральных чисел, что $\sigma(i)=i$ для любого $i>r$. Такая реализация симметрических групп влечет включения $G(r) \subset G\left(r^{\prime}\right)$ при $r \leqslant r^{\prime}$. Через $\operatorname{sgn}(\sigma)$ обозначим знак перестановки $\sigma$. Обозначим также через $\Lambda_{r}^{+}$подмножество множества $\Lambda^{+}$, состоящее из наборов неотрицательных чисел, сумма которых равна $r$. Для любого множества $S$ через $i_{S}$ обозначим тождественное отображение из $S$ в себя.

Рассмотрим произвольное разложение $E^{\otimes r}=\bigoplus_{i=1}^{h_{r}} M_{i}(r)$ в прямую сумму неразложимых $G L_{n}(K)$-модулей. В силу свойств 1) и 2) модуль $E^{\otimes r}$ наклонный. Поэтому в силу свойства 4) и теоремы Крулля-Шмидта модули $M_{i}(r)$ неразложимые наклонные. Положим $A(r)=\operatorname{End}_{G L_{n}(K)} E^{\otimes r}$. Мы имеем естественные проекции $\pi_{i}^{r}: E^{\otimes r} \rightarrow M_{i}(r)$ и вложения $\varepsilon_{i}^{r}: M_{i}(r) \rightarrow E^{\otimes r}$. Положим $e_{i}^{r}=\varepsilon_{i}^{r} \pi_{i}^{r}$. Эндоморфизмы $e_{i}^{r}$ - примитивные идемпотенты и $e_{i}^{r} e_{j}^{r}=0$ при $i \neq j$.

Непосредственно из определения видно, что любой вес модуля $E^{\otimes r}$ имеет неотрицательные компоненты, сумма которых равна $r$. Поэтому имеет место разбиение $\left\{1, \ldots, h_{r}\right\}=\bigsqcup_{\lambda \in \Lambda_{r}^{+}} I_{\lambda}$, где $I_{\lambda}-$ возможно пустые множества чисел и для $i \in I_{\lambda}$ вьполнено $M_{i}(r) \cong P(\lambda)$.

Для каждой пары $i, j \in I_{\lambda}$ выберем некоторый изоморфизм $\tau_{i, j}^{r}$ из $M_{j}(r)$ в $M_{i}(r)$ таким образом, что $\tau_{i, i}^{r}=i_{M_{i}(r)}$ и $\tau_{i, j}^{r} \tau_{j, k}^{r}=\tau_{i, k}^{r}$ для любых $i, j, k \in I_{\lambda}$.

Рассмотрим следующие идемпотенты $e_{\lambda}=\sum_{i \in I_{\lambda}} e_{i}^{r}$. При этом если $I_{\lambda}$ пусто, то положим $e_{\lambda}=0$. Имеет место представление $A(r)=S(r) \oplus J(r)$, где $S(r)-$ множество эндоморфизмов вида

$$
\sum_{\lambda \in \Lambda_{r}^{+}} \sum_{i, j \in I_{\lambda}} \alpha_{i, j} \varepsilon_{i}^{r} \tau_{i, j}^{r} \pi_{j}^{r}
$$


где $\alpha_{i, j} \in K$, а $J(r)$ - множество эндоморфизмов вида

$$
\sum_{i, j=1}^{h_{r}} \varepsilon_{i}^{r} \varphi_{i, j} \pi_{j}^{r}
$$

где $\varphi_{i, j} \in \operatorname{Hom}_{G L_{n}(K)}\left(M_{j}(r), M_{i}(r)\right)$ такие, что если $i, j \in I_{\lambda}$ для некоторого $\lambda \in \Lambda_{r}^{+}$, то эндоморфизм $\varphi_{i, j} \tau_{j, i}^{r}$ нильпотентен. Это разложение легко следует из леммы Фитинга, алгебраической замкнутости поля $K$ и конечномерности всех рассматриваемых модулей. Имеют место следующие формулы

$$
A(r)=\bigoplus_{i, j=1}^{h_{r}} e_{i}^{r} A(r) e_{j}^{r}=\bigoplus_{\lambda \in \Lambda_{r}^{+}} S(r) e_{\lambda} \oplus \bigoplus_{\lambda, \mu \in \Lambda_{r}^{+}} e_{\lambda} J(r) e_{\mu}
$$

При этом $S(r)$ - подалгебра алгебры $A(r)$ и $S(r) e_{\lambda}=e_{\lambda} S(r)=e_{\lambda} S(r) e_{\lambda}$ для любого $\lambda \in \Lambda_{r}^{+}$.

Для $\pi \in G(r)$ рассмотрим элемент $\sigma_{r}(\pi)$ из $A(r)$, действующий по формуле

$$
\sigma_{r}(\pi)\left(v_{i_{1}} \otimes \cdots \otimes v_{i_{r}}\right)=v_{i_{\pi-1}(1)} \otimes \cdots \otimes v_{i^{-1}(r)}
$$

Продолжая действие $\sigma_{r}$ по линейности на $K G(r)$, получаем гомоморфизм из $K G(r)$ в $A(r)$. Пользуясь этим гомоморфизмом, мы будем рассматривать каждый $A$-модуль как $K G(r)$-модуль.

ЛЕмма 1 [10]. Отображсение $\sigma_{r}$ - эпиморфизм. При $r \leqslant n$ отображсение $\sigma_{r}-$ изоморфизм, а при $r \geqslant n+1$ ядро Ker $\sigma_{r}$ порожсдено как двусторонний идеал алгебры $K G(r)$ многочленом $\sum_{\sigma \in K G(n+1)} \operatorname{sgn}(\sigma) \sigma$.

Под разбиением числа $r$ понимаем набор целых чисел $\lambda=\left(\lambda_{1}, \ldots, \lambda_{s}\right)$ такой, что $\lambda_{1} \geqslant \cdots \geqslant \lambda_{s} \geqslant 0$ и $\lambda_{1}+\cdots+\lambda_{s}=r$. При этом этот набор называется $p$-регулярнылм, если никакие $p$ чисел $\lambda_{i}$ не равны некоторому ненулевому числу. Иначе набор называется $p$-сингулярным. Имеется стандартный способ (см. [2]) сопоставления каждому $p$-регулярному разбиению $\lambda$ числа $r$ неприводимого $K G(r)$-модуля $D^{\lambda}$. При этом любой неприводимый $K G(r)$-модуль изоморфен одному из таких $D^{\lambda}$. Используя выше определенный эпиморфизм $\sigma_{r}$, можно каждый неприводимый $K G(r)$-модуль $N$, изоморфиый некоторому $D^{\lambda}$ для $p$-регулярного $\lambda \in \Lambda_{r}^{+}$, превратить в $A(r)$-модуль по формуле $a m=x m$, где $m \in N$, $a \in A(r), x \in K G(r)$ и $\sigma_{r}(x)=a$. Это определение корректно, так как по лемме 1 и непосредственной конструкции $D^{\lambda}$ имеем $\left(\operatorname{Ker} \sigma_{r}\right) D^{\lambda}=0$. Используя лемму 1 , легко проверить, что любой неприводимый $A(r)$-модуль изоморфен одному из $D^{\lambda}$ для некоторого $p$-регулярного $\lambda \in \Lambda_{r}^{+}$.

Будем говорить, что идемпотенту $e_{\lambda}$ соответствует неприводимое представление $D^{\mu}$, если $e_{\lambda} D^{\mu} \neq 0$. При этом очевидно, что $e_{\lambda} m=m$ для любого $m \in D^{\mu}$ и $e_{\nu} D^{\mu}=0$, если $\nu \neq \lambda$. Из общей теории следует, что каждому ненулевому идемпотенту соответствует единственное с точностью до изоморфизма неприводимое представление. 
ЛЕмма 2. Любому идемпотенту $e_{\lambda}$, где $\lambda$ - р-регулярное разбиение из $\Lambda_{r}^{+}$, соответствует представление $D^{\lambda}$. Если $\lambda$-р-сингулярное разбиение, то $e_{\lambda}=0$.

ДоказАТЕЛьство. Для краткости положим $G=G(r), A=A(r), M_{i}=M_{i}(r)$ и $e_{i}=e_{i}^{r}$. Через $X$ обозначим множество таких $\lambda \in \Lambda_{r}^{+}$, что $e_{\lambda} \neq 0$. Для $\lambda \in X$ через $t(\lambda)$ обозначим такое $p$-регулярное разбиение из $\Lambda_{r}^{+}$, что $e_{\lambda} D^{t(\lambda)} \neq 0$. Очевидно, что $t$ - биективное отображение множества $X$ на подмножество $p$-регулярных разбиений множества $\Lambda_{r}^{+}$.

Для любого $\lambda \in \Lambda_{r}^{+}$положим

$$
v(\lambda)=c_{1} \cdots c_{\lambda_{1}} \underbrace{v_{1} \otimes \cdots \otimes v_{1}}_{\lambda_{1} \text { раз }} \otimes \cdots \otimes \underbrace{v_{n} \otimes \cdots \otimes v_{n}}_{\lambda_{n} \text { раз }},
$$

где $c_{i}$ - сумма элементов вида $\operatorname{sgn}(\sigma) \sigma$ по всем $\sigma \in G$ таким, что $\sigma(i)=i$ для любого $i$, не принадлежашего множеству $\left\{i+\sum_{j=1}^{s} \lambda_{j}: 0 \leqslant s \leqslant n-1, i \leqslant \lambda_{s+1}\right\}$. Понятно, что модуль $A v(\lambda)=K G v(\lambda)$ изоморфен как $K G$-модуль модулю Шпехта $S^{\lambda}$, определенному в [2]. Действительно, поставим в соответствие элементу $v_{i_{1}} \otimes \cdots \otimes v_{i_{r}}$ из $\left(E^{\otimes r}\right)^{\lambda}$ таблоид, у которого каждое число $s, s=1, \ldots, r$, стоит в строке $i_{s}$. Такое соответствие, продолженное линейно на все $\left(E^{\otimes r}\right)^{\lambda}$, задает изоморфизм $K G$-модулей $\left(E^{\otimes r}\right)^{\lambda}$ и $M^{\lambda}$. Таблоиды и модуль $M^{\lambda}$ понимаем в смысле определений 3.9 и 4.1 работы [2] с той лиш разницей, что в нашем случае симметрическая группа действует слева. Легко видеть, что ограничение этого изоморфизма на $A v(\lambda)$ дает требуемое соответствие.

Возьмем произвольное $\lambda \in X$. Пусть $P$ - максимальный $A$-подмодуль модуля $A v(t(\lambda))$. Модули $A v(t(\lambda)) / P$ и $D^{t(\lambda)}$ изоморфны как $K G$-модули, а значит, и как $A$-модули. Отсюда и из $e_{\lambda} D^{t(\lambda)} \neq 0$ следует $e_{\lambda} A v(t(\lambda)) \neq 0$ и $e_{i} A v(t(\lambda)) \neq 0$ для некоторого $i \in I_{\lambda}$. Все элементы ненулевого пространства $e_{i} A v(t(\lambda))$ имеют вес $t(\lambda)$. С другой стороны, $e_{i} A v(t(\lambda)) \subset M_{i} \cong P(\lambda)$. Отсюда $t(\lambda)-$ вес модуля $P(\lambda)$ и $t(\lambda) \unlhd \lambda$ в силу свойства 3$)$.

Докажем обратное неравенство. Без ограничения общности можно считать, что $I_{\lambda}=\{1, \ldots, l\}$. Здесь $l \geqslant 1$, так как $e_{\lambda} \neq 0$. Известно, что модуль $K G L_{n}(K) v(\lambda)$ изоморфен так называемому модулю Вейля со старшим весом $\lambda$. Но этот же модуль вкладывается в $P(\lambda)$. Поэтому сушествует вложение $G L_{n}(K)$-модулей $\tau: K G L_{n}(K) v(\lambda) \rightarrow M_{1}$. Пусть $v=\tau(v(\lambda))$.

Пусть $U$ - подгруппа группы $G L_{n}(K)$, порожденная такими автоморфизмами $\varphi$, что $\varphi\left(v_{k}\right)=v_{k}$ при $k \neq i$ и $\varphi\left(v_{i}\right)=v_{i}+\alpha v_{j}$, где $\alpha \in K$ и $j<i$. Следствие 17.18 работы [2] фактически означает, что элемент $m \in\left(E^{\otimes r}\right)^{\lambda}$ принадлежит $A v(\lambda)$ тогда и только тогда, когда $u m=m$ для любого $u \in U$. Используя этот факт и то, что $\tau$ - вложение $G L_{n}(K)$-модулей, мы получаем $v \in A v(\lambda)$.

Если бы идемпотент $e_{\lambda}$ аннулировал любой композиционный фактор модуля $A v$, то мы бы имели $e_{\lambda} A v=\left(e_{\lambda}\right)^{s} A v=0$. Однако $v \in e_{\lambda} A v$. Поэтому $e_{\lambda} D^{\mu} \neq 0$, где $D^{\mu}$ - композиционный фактор модуля $A v$, а следовательно, и модуля $A v(\lambda)$. Так как $A v(\lambda) \cong S^{\lambda}$, то $\mu \unrhd \lambda$. По определению $\mu=t(\lambda)$ и, следовательно, по ранее доказанному $\mu \unlhd \lambda$. Отсюда мы получаем $t(\lambda)=\lambda$ и $A v=A v(\lambda)$. Лемма доказана.

В процессе доказательства мы одновременно получили 
СЛЕДСТВИЕ 1. Пусть $v$ - ненулевой әлемент из $M_{i} \cong P(\lambda)$ веса $\lambda$. Тог-

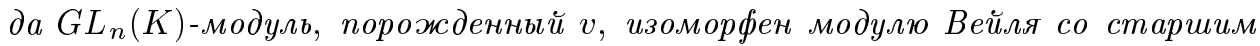
весом $\lambda$, a $G(r)$-модуль, порохсденный $v$, изоморфен модулю Шпехта $S^{\lambda}$.

Лемма 2 очень важна для нашей теории. Она дает описание через примитивные идемпотенты алгебры $A$ тех же самых неприводимых модулей, которые описаны в [2] и которые изучались в [1]. Без нее выше описанный подход был бы неэффективен. Автору не известно, как точно сослаться на это утверждение. Поэтому здесь приведено его доказательство.

\section{§ 3. Операторы вложения}

В предыдущем параграфе мы выбрали произвольное разложение модуля $E^{\otimes r}$ в прямую сумму неразложимых наклонных модулей. Однако нам будет удобно, чтобы такие разложения для разных $r$ были согласованы. Поэтому далее мы будем строить их по индукции следующим образом.

Сначала положим $M_{1}(1)=E$. Пусть имеется разложение

$$
E^{\otimes r}=\bigoplus_{i=1}^{h_{r}} M_{i}(r)
$$

В силу свойств 1$)$ и 2) каждый модуль $M_{i}(r) \otimes E$ наклонный. Поэтому по свойству 4) он разложим в прямую сумму неразложимых наклонных модулей. Собирая все такие слагаемые, получаем разложение модуля $E^{\otimes r+1}$.

Пусть $r<r^{\prime}$ два натуральных числа. Определим оператор вложения

$$
f_{r^{\prime}, r}: A(r) \rightarrow A\left(r^{\prime}\right)
$$

по формуле $f_{r^{\prime}, r}(\varphi)=\varphi \otimes i_{E \otimes r^{\prime}-r}$. В этом случае имеем $f_{r^{\prime}, r} \circ \sigma_{r}=\left.\sigma_{r^{\prime}}\right|_{K G(r)}$ и $f_{r^{\prime \prime}, r^{\prime}} \circ f_{r^{\prime}, r}=f_{r^{\prime \prime}, r}$. Понятно, что если $M_{i}(r) \otimes E^{\otimes r^{\prime}-r}=\bigoplus_{j \in X} M_{j}\left(r^{\prime}\right)$, то $f_{r^{\prime}, r}\left(e_{i}^{r}\right)=\sum_{j \in X} e_{j}^{r^{\prime}}$

Напомним терминологию и обозначения, использованные в [1]. Разбиение $\lambda \in \Lambda_{r}^{+}$назьвается вырожденным $(n$-вырожденным $)$, если $\lambda_{n}=0$. Для двух разбиений $\lambda, \mu \in \Lambda_{r}^{+}$и целых $\alpha, \beta$ через $\alpha \lambda+\beta \mu$ обозначаем покомпонентную линейную комбинацию этих разбиений. Через $\left(i^{n}\right)$ обозначаем набор длины $n$, в котором все компоненты равны $i$. Через $d_{r}$ обозначаем сумму идемпотентов $e_{\lambda}$ по всем вырожденньм $\lambda \in \Lambda_{r}^{+}$.

Предположим теперь, что $\lambda$ - разбиение из $\Lambda_{r}^{+}$, для которого вьполнено

УСЛОВИЕ 1. Существует а такое, что для любого вырожсденного $\mu \in$ $\Lambda_{r+a n}^{+} u k \geqslant a$ умодуля $P(\mu) \otimes E^{\otimes(k-a) n}$ нет прямого слагаемого, изоморфного $P\left(\lambda+\left(k^{n}\right)\right)$.

Отсутствие выше упомянутого прямого слагаемого для любого вырожденного $\mu$ эквивалентно тому, что $e_{\lambda+\left(k^{n}\right)} f_{r+k n, r+a n}\left(d_{r+a n}\right)=0$. Как мы увидим в $\S 4$, такое предположение не лишено смысла. 
ТЕорема 1. Предположим, что разбиение $\lambda+\left(1^{n}\right)$ р-регулярное. Пусть $k \geqslant r^{2} / n+(2 r+1) a+a^{2} n$ и $\mu$ - вырожсденное $p$-регулярное разбиение из

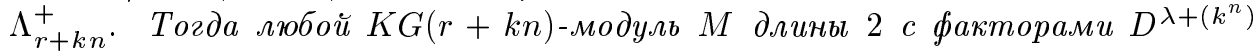
и $D^{\mu}$ разложим.

ДокАЗАТЕльство. Переходя, если необходимо, к двойственным модулям, можно без ограничения общности считать, что существует такой подмодуль $N \subset M$, что $N \cong D^{\mu}$ и $M / N \cong D^{\lambda+\left(k^{n}\right)}$.

Пусть $x$ и $y$ такие элементы из $K G(r+a n)$, что $\sigma_{r+a n}(x)=1-d_{r+a n}$ и $\sigma_{r+a n}(y)=d_{r+a n}$. При этом $\sigma_{r+k n}(x)=f_{r+k n, r+a n}\left(1-d_{r+a n}\right)$ и $\sigma_{r+k n}(y)=$ $f_{r+k n, r+a n}\left(d_{r+a n}\right)$.

Имеют место следующие равенства:

$$
\begin{array}{ll}
\sigma_{r+k n}(y) e_{\lambda+\left(k^{n}\right)}=0, & \sigma_{r+k n}(x) e_{\mu}=0, \\
\sigma_{r+k n}(y) e_{\mu}=e_{\mu}, & \sigma_{r+k n}(x) e_{\lambda+\left(k^{n}\right)}=e_{\lambda+\left(k^{n}\right)} .
\end{array}
$$

Первое из них следует из сделанного предположения о $\lambda$. Второе следует из того, что для любого невырожденного $\nu \in \Lambda_{r+a n}^{+}$у модуля $P(\nu) \otimes E^{\otimes(k-a) n}$ нет прямого слагаемого, изоморфного $P(\mu)$. Последние два равенства следуют из уже доказанных и формулы $\sigma_{r+k n}(x)+\sigma_{r+k n}(y)=1$.

Рассмотрим сдвиги элемента $x$, определенные формулой $x_{i}=\pi_{i} x \pi_{i}^{-1}$, $i=1, \ldots, r+a n+1$, где $\pi_{i}=\prod_{j=1}^{r+a n}(j+(i-1)(r+a n), j)$. Заметим, что $\operatorname{supp}\left(x_{i}\right)=\{1+(i-1)(r+a n), \ldots, i(r+a n)\}$. Так как $\operatorname{supp}\left(x_{i}\right)$ для различных $i$ не пересекаются, то элементы $x_{i}$ коммутируют. Ограничение $k$ снизу в условии теоремы обеспечивает принадлежность всех $x_{i}$ алгебре $K G(r+k n)$.

Имеют место соотношения

$$
y M \subset N, \quad x_{i} N=0, \quad y N \neq 0,
$$

которые следуют из первых трех равенств (1) и леммы 2.

Выберем произвольный элемент $m \in M \backslash N$ и докажем, что справедливо соотношение $x_{1} \cdots x_{r+a n+1} m \notin N$. Предположим противное. Тогда

$$
\sigma_{r+k n}\left(x_{1}\right) \cdots \sigma_{r+k n}\left(x_{r+a n+1}\right) \bar{m}=0,
$$

где $\bar{m}$ - образ $m$ при проекции $M \rightarrow M / N$. Отсюда, используя последнюю из формул (1) и лемму 2 , получаем

$$
\begin{aligned}
\sigma_{r+k n}\left(x_{i}\right) \bar{m} & =\sigma_{r+k n}\left(\pi_{i}\right) \sigma_{r+k n}(x) \sigma_{r+k n}\left(\pi_{i}^{-1}\right) \bar{m} \\
& =\sigma_{r+k n}\left(\pi_{i}\right) \sigma_{r+k n}(x) e_{\lambda+\left(k^{n}\right)} \sigma_{r+k n}\left(\pi_{i}^{-1}\right) \bar{m}=\bar{m} .
\end{aligned}
$$

В результате $\bar{m}=0$. Приходим к противоречию.

Пусть $P-K G(r+k n)$-модуль, порожденный элементом $x_{1} \cdots x_{r+a n+1} m$. Вычислим, чему равно $y P$. Возьмем $\alpha \in G(r+k n)$. Мощность множества $\operatorname{supp}\left(\alpha^{-1} y \alpha\right)$ не превосходит $r+a n$. Поэтому существует $i=1, \ldots, r+a n+1$ такое, что элементы $\alpha^{-1}$ у $\alpha$ и $x_{i}$ коммутируют. Имеем

$$
y \alpha x_{1} \cdots x_{r+a n+1} m \in \alpha x_{i}\left(\alpha^{-1} y \alpha\right) M \subset \alpha x_{i} N=0 .
$$

Отсюда $y P=0$. Так как $y N \neq 0$, то $P \cap N=0$ и $M=P \oplus N$. Теорема доказана.

Индукцией по длине легко доказать, что в условиях теоремы 1 любой $K G(r+k n)$-модуль $M$ конечной длины, у которого один фактор изоморфен $D^{\lambda+\left(k^{n}\right)}$, а остальные вырождены, содержит подмодуль, изоморфньй $D^{\lambda+\left(k^{n}\right)}$. 


\section{§4. Примеры}

Мы доказали теорему 1 в предположении, что $\lambda$ удовлетворяет условию 1. В этом параграфе мы приведем два примера таких разбиений.

ПримеР 1. Предположим, что $n<p$. Для любого натурального $R$ положим $C_{0}(R)=\left\{\lambda \in \Lambda_{R}^{+}: \lambda_{1}-\lambda_{n} \leqslant p-n\right\}$. Лемма 12(2) работы [5] утверждает, что если $\mu \in \Lambda_{R}^{+} \backslash C_{0}(R)$, то у модуля $P(\mu) \otimes E$ нет прямого слагаемого, изоморфного $P(\nu)$, где $\nu \in C_{0}(R+1)$. Отсюда очевидно следует, что для любого $i$ у модуля $P(\mu) \otimes E^{i}$ нет прямого слагаемого, изоморфного $P(\nu)$, где $\nu \in C_{0}(R+i)$.

Выберем произвольное $\lambda$ из $C_{0}(r)$ и докажем, что оно удовлетворяет условию 1 .

Лемма 3. Пусть $R \geqslant(n-1)(p-n)+1$ и $\Lambda_{R}^{+}$. Тогда $\mu \notin C_{0}(R)$.

ДокаЗАтельство. Имеем $(n-1) \mu_{1} \geqslant \mu_{1}+\cdots+\mu_{n-1}=R>(n-1)(p-n)$. Отсюда $\mu_{1}-\mu_{n}=\mu_{1}>p-n$ и $\mu \notin C_{0}(R)$. Лемма доказана.

Выберем некоторое, например, минимальное $a$, удовлетворяющее неравенству $r+a n \geqslant(n-1)(p-n)+1$. Тогда в силу леммы 12(2) работы [5] и леммы 3 разбиение $\lambda$ удовлетворяет условию 1 для данного $a$. Следовательно, к разбиению $\lambda$ можно применить теорему 1 . Однако учитывая специфику случая $n<p$, возможно улучшить оценку этой теоремы.

Tеорема 2. Пусть $\lambda \in C_{0}(r), \lambda_{n} \geqslant(n-1)(p-n)+2 u \mu-$ вырожсденное разбиение из $\Lambda_{r}^{+}$. Тогда любой $K G(r)$-модуль $M$ длины 2 с факторами $D^{\lambda}$ и $D^{\mu}$ разложим.

ДокАЗАТЕЛЬСТво отличается от доказательства теоремы 1 в основном выбором элементов $x$ и $y$. Аналогично теореме 1 без ограничения общности можно считать, что сушествует такой подмодуль $N \subset M$, что $N \cong D^{\mu}$ и $M / N \cong D^{\lambda}$. Для краткости положим $R=(n-1)(p-n)+1$.

Пусть $x=(1 / n !) \sum_{\sigma \in G(n)} \operatorname{sgn}(\sigma) \sigma$ и $x_{i}=\pi_{i} g \pi_{i}^{-1}, i=1, \ldots, R+1$, где $\pi_{i}=\prod_{j=1}^{n}(j+(i-1) n, j)$. Выберем $y \in K G(R)$ так, чтобы $\sigma_{R}(y)=d_{R}$. Тогда $\sigma_{r}(y)=f_{r, R}\left(d_{R}\right)$. По лемме $12(2)$ работы [5] и лемме 3 тогда получаем $\sigma_{r}(y) e_{\lambda}=0$. Первая и третья из формул (2) проверяются так же, как в теореме 1. Вторая формула следует из того, что $\mu$ содержит не более $n-1$ нулевых частей. Из непосредственной конструкции модуля $D^{\lambda}$ как фактормодуля модуля Шпехта $S^{\lambda}$ и неравенства $\lambda_{n} \geqslant R+1$ следует, что существует $m \in M$, для которого $x_{1} \cdots x_{R+1} m \notin N$. Через $P$ обозначим $K G(r)$-подмодуль модуля $M$, порожденный элементом $x_{1} \cdots x_{R+1} m$, и аналогично теореме 1 покажем, что $y P=0$. Отсюда $M=P \oplus N$. Теорема доказана.

Пример 2. Предположим, что $n=2$. Выберем произвольное $\lambda \in \Lambda_{r}^{+}$. Пусть $\mu \in \Lambda_{r+1}^{+}$такое, что модуль $P(\mu)$ есть прямое слагаемое модуля $P(\lambda) \otimes E$. Предположим, что $k$ - некотороенатуральное число, для которого $\lambda_{1}-\lambda_{2} \geqslant p^{k}-1$. Докажем, что тогда $\mu_{1}-\mu_{2} \geqslant p^{k}-1$. Доказательство будет заключаться в применении формул для произведения наклонных модулей $\S 1$ работы [6]. В этих формулах речь идет о наклонных модулях над $S L_{2}(K)$. Однако понятно, что если вместо верной 
формулы вида $T(s) \otimes T(1)=T\left(s^{\prime}\right)$ мы напишем

$$
P\left(\left(\frac{r+s}{2}, \frac{r-s}{2}\right)\right) \otimes E=P\left(\left(\frac{r+1+s^{\prime}}{2}, \frac{r+1-s^{\prime}}{2}\right)\right),
$$

то опять получим верную формулу. Будем иметь в виду такие переформулировки при цитировании формул из работы [6] в ниже приведенных вычислениях.

Положим для краткости $m=\lambda_{1}-\lambda_{2}$ и $m^{\prime}=\mu_{1}-\mu_{2}$. Будем считать, что $m>0$ и $k>0$, так как иначе доказываемое утверждение очевидно. Пусть $m=\sum_{s=0}^{\infty} i_{s} p^{s}$, где $0 \leqslant i_{s} \leqslant p-1,-$ разложение числа $m$ по основанию $p$.

Если $i_{0}=p-1$, то по лемме $1.5(1)$ работы [6] получаем $m^{\prime}=m+1 \geqslant p^{k}$. Если $0 \leqslant i_{0} \leqslant p-3$ (случай $p>2$ ), то $m \geqslant p^{k}$. По лемме $1.5(2),(3)$ работы [6] получаем, что $m^{\prime}=m+1$ или $m^{\prime}=m-1$. В обоих случаях $m^{\prime} \geqslant p^{k}-1$.

Предположим теперь, что $i_{0}=p-2$. Через $t$ обозначим такое положительное число, что $i_{s}=p-1$ для $s=1, \ldots, t-1$ и $i_{t}<p-1$. Положим $\sigma=$ $\sum_{s=t+1}^{\infty} i_{s} p^{s-t-1}$. Тогда $m+2=\left(i_{t}+1\right) p^{t}+\sigma p^{t+1}$.

Случай $p>2$. Для вычислений будем применять лемму 1.7.3 работы [6]. Для доказательства неравенства $m^{\prime} \geqslant p^{k}-1$ достаточно проверить, что $m+1-$ $2 p^{t-1} \geqslant p^{k}-1$ и что $m+1-2 p^{t} \geqslant p^{k}-1$ в случае, когда $i_{t}=1$ и $\sigma>0$ или когда $i_{t} \geqslant 2$.

Предположим, что $t<k$. Из того что $m \geqslant p^{k}$, получаем $i_{k^{\prime}}>0$ для некоторого $k^{\prime} \geqslant k$. Отсюда $m+2 \geqslant\left(i_{t}+1\right) p^{t}+p^{k}$ и $m+1-2 p^{t-1} \geqslant p^{k}-1+p^{t}-2 p^{t-1}>p^{k}-1$. Если $i_{t} \geqslant 1$, то $m+1-2 p^{t} \geqslant p^{k}-1+\left(i_{t}-1\right) p^{t} \geqslant p^{k}-1$.

Предположим, что $t=k$. Из того что $m \geqslant p^{k}$, получаем либо $i_{k}>0$ и $\sigma=0$, либо $\sigma>0$. В первом случае $m+2 \geqslant 2 p^{k}$ и $m+1-2 p^{t-1} \geqslant 2 p^{k}-1-2 p^{k-1}>p^{k}-1$. При этом если $i_{k} \geqslant 2$, то $m+2 \geqslant 3 p^{k}$ и $m+1-2 p^{t} \geqslant p^{k}-1$. Во втором случае $m+2 \geqslant 3 p^{k}$ и $m+1-2 p^{t} \geqslant p^{k}-1$.

Предположим, что $t>k$. Так как $m+2 \geqslant p^{t}$, то $m+1-2 p^{t-1} \geqslant p^{t-1}-1 \geqslant p^{k}-1$. Если $\sigma>0$ или $i_{t} \geqslant 2$, то $m+2 \geqslant 3 p^{t}$ и $m+1-2 p^{t} \geqslant p^{t}-1>p^{k}-1$.

Случай $p=2$. Для вычислений будем применять лемму 1.7 .2 работы [6]. В данном случае для доказательства неравенства $m^{\prime} \geqslant 2^{k}-1$ достаточно проверить, что $m+1-2^{t-1} \geqslant 2^{k}-1$ и что $m+1-2^{t} \geqslant 2^{k}-1$ в случае $\sigma>0$.

Предположим, что $t<k$. Аналогично случаю $p>2$ получаем $m+2 \geqslant 2^{t}+2^{k}$. Отсюда $m+1-2^{t} \geqslant 2^{k}-1$.

Предположим, что $t=k$. Аналогично случаю $p>2$ получаем либо $i_{k}>0$, либо $\sigma>0$. В обоих случаях $m+2 \geqslant 2^{k+1}$ и $m+1-2^{t} \geqslant 2^{k+1}-1-2^{k}=2^{k}-1$.

Предположим, что $t>k$. Так как $m+2 \geqslant 2^{t}$, то $m+1-2^{t-1} \geqslant 2^{t-1}-1 \geqslant 2^{k}-1$. Если $\sigma>0$, то $m+2 \geqslant 2^{t+1}$ и $m+1-2^{t} \geqslant 2^{t}-1>2^{k}-1$.

Теперь можно проверить выполнение условия 1 для любого разбиения $\lambda \in \Lambda_{r}^{+}$. Выберем некоторое, например, минимальное $k$ такое, что $\lambda_{1}-\lambda_{2}<p^{k}-1$, и положим $a=p^{k}-1-r$. Из выше приведенных вычислений видно, что для таким образом выбранного $а$ условие 1 выполнено.

\section{§5. Конечная базируемость}

Посмотрим, какие следствия из результатов $\S 4$ можно получить применительно к операции $\uparrow$, введенной в [1]. 
Для любого $K G(r)$-модуля $V$ через $\mathrm{d}(V)$ обозначим модуль, дуальный к $V$. При этом сушествует такая невырожденная форма $\langle\cdot, \cdot\rangle: V \times \mathrm{d}(V) \rightarrow K$, что $\langle\sigma v, u\rangle=$ $\left\langle v, \sigma^{-1} u\right\rangle$ для любых $v \in V, u \in \mathrm{d}(V)$ и $\sigma \in G(r)$. Для подмножества $A$ в $V$ или в $\mathrm{d}(V)$ через $V^{\perp}$ будем обозначать множество $\{u \in \mathrm{d}(V):\langle A, u\rangle=0\}$ или $\{v \in V:\langle v, A\rangle=0\}$ соответственно.

Лемма 4. Пусть $\lambda$-р-регулярное невырожсденное разбиение из $\Lambda_{r}^{+} u V-$ собственный подмодуль модуля ШПехта $S^{\lambda}$. Рассмотрим следующие условия:

1) $V \downarrow \uparrow \varsubsetneqq V$;

2) существует $K G(r)$-модуль $L$ и неизоморфное влохсение $\iota: \mathrm{d}\left(S^{\lambda} / V\right) \rightarrow L$ такое, что $\operatorname{Im} \iota$ - существенный подмодуль модуля $L$ и все факторь модуля L/ Im $\iota$ вырохсденные.

Условие 2) всегда следует из 1). Обратное следствие верно при $n<p$.

ДокАЗАТЕльство. 1$) \Rightarrow 2)$ Положим $L=\mathrm{d}\left(S^{\lambda} / V \downarrow \uparrow\right)$ и $\iota$ равным вложению, индуцированному проекцией $S^{\lambda} / V \downarrow \uparrow \rightarrow S^{\lambda} / V$. Из очевидного равенства $V \downarrow \uparrow \downarrow=$ $V \downarrow$ (см. $\S 1)$ и теоремы $5($ a) работы [1] следует, что все факторы модуля $V / V \downarrow \uparrow$ вырожденные. Поэтому все факторы модуля $L / \operatorname{Im} \iota$ тоже вырожденные. Осталось доказать, что модуль $\operatorname{Im} \iota$ сушественный в $L$. Пусть $N$ - подмодуль модуля $L$ и $N \cap \operatorname{Im} \iota=0$. Тогда $N^{\perp}+(V / V \downarrow \uparrow)=S^{\lambda} / V \downarrow \uparrow$. Так как $P^{\lambda} / V \downarrow \uparrow-$ единственный максимальный подмодуль модуля $S^{\lambda} / V \downarrow \uparrow$ и $V \subset P^{\lambda}$, то $N^{\perp}=S^{\lambda} / V \downarrow \uparrow$. Отсюда $N=0$.

$2) \Rightarrow 1)$ при условии $n<p$. Без ограничения общности можно считать, что $L / \operatorname{Im} \iota \cong D^{\mu}$, где $\mu$ - вырожденное $p$-регулярное разбиение.

В оставшейся части доказательства мы будем следовать обозначениям работы [11]. Выберем произвольное $N \geqslant r$ и положим $G=G(r), S=S_{K}(N, r)$, $\omega=\left(1^{r}, 0^{N-r}\right), e=\xi_{\omega}$. Фиксируем набор $u(r)=(1, \ldots, r)$. Отображение $\xi_{u(r) \pi, u(r)} \mapsto \pi$ задает изоморфизм между кольцами $e S e$ и $K G$. Поэтому мы будем рассматривать каждый $K G$-модуль как $e S e$-модуль и наоборот.

Пусть $V_{\lambda^{\prime}, K}$ - модуль Вейля со старшим весом $\lambda^{\prime}$. В работе [11] при помоши изоморфизма (6.3d) устанавливается изоморфизм модулей $V_{\lambda^{\prime}, K}^{\omega}$ и $\mathrm{d}\left(S^{\lambda^{\prime}}\right)\left(\bar{S}_{T, K}\right.$ в обозначениях [11]).

Через $K_{s}$ обозначим знакопеременное представление группы $G$. Модуль $V \otimes K_{s}$ стандартно вкладывается в $\mathrm{d}\left(S^{\lambda^{\prime}}\right)$ и, следовательно, в $V_{\lambda^{\prime}, K}^{\omega}$. Пусть $V^{\prime}-$ образ $V$ при таком вложении.

Положим $U=S V^{\prime}$. Этот модуль является подмодулем модуля $V_{\lambda^{\prime}, K}$. В paботе [11] введены левые $S$-модули $D_{\lambda^{\prime}, K}$ и $A_{K}(N, r)$. Последний модуль - также правый $S$-модуль. При этом $D_{\lambda^{\prime}, K}$ - подмодуль модуля ${ }^{\lambda^{\prime}} A_{K}(N, r)$, который является правьм весовым пространством модуля $A_{K}(N, r)$.

Согласно $\S 5.1$ работы [11] ограничение естественной формы $\langle\cdot, \cdot\rangle$, определенной на $E^{\otimes r}$, дает невырожденную контравариантную форму $(\cdot, \cdot): V_{\lambda^{\prime}, K} \times D_{\lambda^{\prime}, K} \rightarrow K$. Положим $W=\left\{w \in D_{\lambda^{\prime}, K}:(U, w)=0\right\}$. Модуль $W$ контравариантно дуален модулю $V_{\lambda^{\prime}, K} / U$ в смысле $\S 2.7$ работы [11]. Поэтому модуль $e W$ двойствен модулю $V_{\lambda^{\prime}, K}^{\omega} / V^{\prime}$ как $K G$-модуль в обычном смысле (см. [11; теорема 6.3f]). При этом $V_{\lambda^{\prime}, K}^{\omega} / V^{\prime} \cong S^{\lambda} / V \otimes K_{s}$. Отсюда получаем $e W \cong \mathrm{d}\left(S^{\lambda} / V\right) \otimes K_{s}$. Рассмот- 
рим некоторьй изоморфизм $\theta: e W \rightarrow \mathrm{d}\left(S^{\lambda} / V\right) \otimes K_{s}$. Положим $M=L \otimes K_{s}$ и $\varphi=\left(\iota \otimes i_{K_{s}}\right) \theta$. Понятно, что $\varphi-$ вложение модуля $e W$ в $M$. При этом $\operatorname{Im} \varphi-$ сушественньй подмодуль модуля $M$ и $M / \operatorname{Im} \varphi \cong D^{\mu} \otimes K_{s}$.

Положим $h(M)=S e \otimes_{e S e} M$. Через $W^{\prime}$ обозначим $K$-подпространство пространства $h(M)$, порожденное элементами вида $s e \otimes \varphi(e w)$, где $s \in S$ и $w \in W$. Очевидно, что $W^{\prime}$ есть $S$-модуль. Рассмотрим отображение $\psi: W^{\prime} \rightarrow W$, определенное формулой $\psi(s e \otimes \varphi(e w))=s e w$.

Так как $W \subset \lambda^{\prime} A_{K}(N, r)$ и последний модуль инъективен, то сушествует продолжение $\bar{\psi}: h(M) \rightarrow{ }^{\lambda^{\prime}} A_{K}(N, r)$ отображения $\psi$. Рассмотрим изоморфизм $\varepsilon: M \rightarrow e h(M)$, заданный формулой $\varepsilon(m)=e \otimes m$. Пусть $\tau=\bar{\psi} \varepsilon-$ их композиция. Понятно, что $\tau \varphi=i_{e W}$ Следовательно, $\tau$ изоморфно отображает $\operatorname{Im} \varphi$ на $e W$. Так как $\operatorname{Im} \varphi$ - существенный подмодуль модуля $M$, то $\tau$ - вложение $M$ в $e^{\lambda^{\prime}} A_{K}(N, r)$.

Модуль $e^{\lambda^{\prime}} A_{K}(N, r)$ можно отождествить с модулем $M^{\lambda^{\prime}}$ в обозначениях работы [2]. При этом модулю $e D_{\lambda^{\prime}, K}$ будет соответствовать модуль Шпехта $S^{\lambda^{\prime}}$. Применяя результаты $\S 17$ работы [2], получаем, что сушествует фильтрация модуля $e^{\lambda^{\prime}} A_{K}(N, r) / e D_{\lambda^{\prime}, K}$ с факторами, изоморфньми $S^{\nu}$, где $\nu \triangleright \lambda^{\prime}$. Поэтому либо $\tau(M) \subset e D_{\lambda^{\prime}, K}$, либо некоторый модуль $S^{\nu}$, где $\nu \triangleright \lambda^{\prime}$, содержит подмодуль, изоморфный $D^{\mu} \otimes K_{s}$.

Покажем, что последний случай невозможен. Так как $S^{\nu} \subset M^{\nu}$ (см. [2]) и модули $D^{\mu} \otimes K_{s}$ и $M^{\nu}$ самодвойственные, то сушествует эпиморфизм $\pi: M^{\nu} \rightarrow D^{\mu} \otimes K_{s}$. Положим $g=\sum_{\sigma \in G(n)} \sigma$. Так как $\nu \triangleright \lambda^{\prime}$, то $\nu_{1} \geqslant n$. Отсюда и из неравенства $n<p$ получаем, что у модуля $M^{\nu}$ существует циклическая порождающая вида $g v$, где $v \in M^{\nu}$. Применяя $\pi$, получаем $\pi(g v)=g \pi(v) \in g\left(D^{\mu} \otimes K_{s}\right)=0$. Последнее равенство следует из вырожденности $\mu$. Приходим к противоречию.

Итак, мы получили $e W \subset \tau(M) \subset e D_{\lambda^{\prime}, K}$. Применяя форму $(\cdot, \cdot)$ и умножая на $K_{s}$, получаем, что сушествует некоторый подмодуль $V_{0}$ модуля $V$ такой, что $V / V_{0} \cong D^{\mu}$. В силу следствия 6 работы [1] имеем $V \downarrow \uparrow \varsubsetneqq V$. Лемма доказана.

ЗАмЕчАнИЕ. В первой части доказательства достаточно считать, что поле любое, а во второй - бесконечное. Впрочем, вторая часть приведена только для иллюстрации связи свойств 1) и 2) и нигде в данной работе не применяется.

Для любого $p$-регулярного разбиения $\nu$ через $P^{\nu}$ обозначим единственный максимальный подмодуль модуля Шпехта $S^{\nu}$. Легко проверить, что если $\nu$ невырожденное, то $P^{\nu} \downarrow=P^{\nu-\left(1^{n}\right)}$. Теперь в силу первой части леммы 4 получаем, что в условиях теорем 1 и 2 выполнены равенства

$$
P^{\lambda+\left(k-1^{n}\right) \uparrow}=P^{\lambda+\left(k^{n}\right)}, \quad P^{\lambda-\left(1^{n}\right) \uparrow}=P^{\lambda}
$$

соответственно. Если поле $K$ не алгебраически замкнуто, то, применяя естественный изоморфизм $S_{K}^{\nu} \otimes \bar{K} \cong S \frac{\nu}{K}$, при котором $P_{K}^{\nu} \otimes \bar{K}$ переходит в $P \bar{K}$, получаем справедливость формул (3) для любого поля той же характеристики. Из этих формул следует, что для $\lambda$, удовлетворяющего условию 1 , существует конеч-

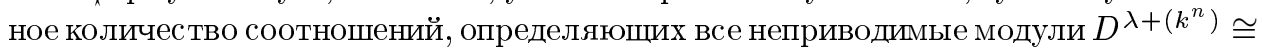
$S^{\lambda+\left(k^{n}\right)} / P^{\lambda+\left(k^{n}\right)}, k \in \mathbb{N}$. 


\section{Список литературы}

1. Shchigolev $V . V$. On the stabilization problem for submodules of Specht modules // J. Algebra. 2002. V. 251. № 2. P. 790-812.

2. Джсеймс Г. Теория представлений симметрической группы. М.: Мир, 1980.

3. Kleshchev A.S. Branching rules for modular representations of symmetric groups. I // J. Algebra. 1995. V. 178. № 2. P. 493-511.

4. Kleshchev A.S. Branching rules for modular representations of symmetric groups. II // J. Reine Angew. Math. 1995. V. 459. P. 163-212.

5. Mathieu $O$. On the dimension of somemodular irreducible representations of the symmetric group // Lett. Math. Phys. 1996. V. 38. №1. P. 23-32.

6. Erdmann $K$. Tensor products and dimensions of simple modules for symmetric groups // Manuscripta Math. 1995. V. 88. № 3. P. 357-386.

7. Donkin S. On tilting modules for algebraic groups // Math. Z. 1993. V. 212. № 1. P. 39-60.

8. Ringel C. M. The category of modules with good filtrations over a quasi-hereditary algebra has almost split sequences // Math. Z. 1991. V. 208. № 2. P. 209-223.

9. Mathieu O. Filtrations of $G$-modules // Ann. Sci. École Norm. Sup. (4). 1990. V. 23. № 4 . P. 625-644.

10. De Concini C., Procesi C. A characteristic free approach to invariant theory // Adv. Math. 1976. V. 21. № 3. P. 330-354.

11. Green J. A. Polynomial representations of $G L_{n}(K)$. Berlin: Springer-Verlag, 1980. (Lecture Notes in Math. V. 830.)

Московский государственнњй

Поступила в редакцию

университет им. М.В. Ломоносова

06.05 .2002 